\title{
A GENERALIZATION OF THE POLAR REPRESENTATION OF NONSINGULAR MATRICES
}

\author{
JOHN WILLIAMSON
}

1. Introduction. If $A$ is a square matrix with elements in the complex number field, then

$$
A=P U,
$$

where $P$ is a positive definite hermitian matrix and $U$ is a unitary matrix. ${ }^{1}$ In this polar representation of the matrix $A$, as it is called, the two matrices $P$ and $U$ are unique. Since the matrix $P$ is positive definite and nonsingular, it has the same signature as the identity matrix $E$ while the unitary matrix $U$ is a conjunctive automorph of $E$. From (1) we may deduce a somewhat similar representation of $A$ in terms of a positive definite hermitian matrix and a conjunctive automorph, not of $E$, but of any nonsingular positive definite hermitian matrix.

Let $H$ be a nonsingular hermitian matrix which is positive definite, so that there exists a nonsingular matrix $Q$ satisfying

$$
Q^{-1} H\left(Q^{-1}\right)^{*}=E,
$$

where $Q^{*}$ is the conjugate transposed of $Q$. If $B=Q^{-1} A Q$ and $B=P U$ is the polar representation of $B$, then $A=Q P Q^{-1} Q U Q^{-1}=D R$, where $D=Q P Q^{-1}$ and $R=Q U Q^{-1}$. Since $D H=Q P Q^{-1} Q Q^{*}=Q P Q^{*}, D H$ is hermitian with the same signature as $H$. Further $R H R^{*}=Q U Q^{-1} Q Q^{*}$ $\cdot\left(Q^{-1}\right)^{*} U^{*} Q^{*}=Q Q^{*}=H$. Hence we have this result as an analogue of the polar representation (1) of $A$.

Result (1). If $H$ is any nonsingular positive definite hermitian matrix and $A$ is a nonsingular matrix, then

$$
A=D R,
$$

where $D H$ is a positive definite hermitian matrix and $R H R^{*}=H$.

If $H=H^{-1}, A=D R=D H H R$ and, since $H R H(H R)^{*}=H^{3}=H$, $A=P_{1} R_{1}$, where $P_{1}$ is a positive definite hermitian matrix and $R_{1} H R_{1}{ }^{*}=H$. Therefore we have as a second analogue of (1) the following result.

Received by the editors October 13, 1941.

${ }^{1}$ L. Autonne, Sur les groupes linéaires, réels et orthogonaux, Bulletin de la Société Mathématique de France, vol. 30 (1902), pp. 121-134. A. Wintner and F. D. Murnaghan, On a polar representation of non-singular matrices, Proceedings of the National Academy of Sciences, vol. 17 (1931), pp. 676-678. 
REsult (2). If $H$ is a positive definite hermitian matrix, whose square is the identity matrix, and, if $A$ is a nonsingular matrix, then

$$
A=P_{1} R_{1},
$$

where $P_{1}$ is a positive definite hermitian matrix and $R_{1}$ is a conjunctive automorph of $H$. The two matrices $P_{1}$ and $R_{1}$ are unique.

It is our intention here to determine what corresponds to results (1) and (2), if $H$ is a nonsingular hermitian matrix but not necessarily definite.

2. Generalization in the complex field. Let $H$ be any nonsingular hermitian matrix and let $A$ be a nonsingular matrix. If

$$
A=D R \text {, }
$$

where

$$
D H=H D^{*}
$$

and

$$
R H R^{*}=H,
$$

we shall call (4) a polar representation of $A$ with respect to $H$ or, for brevity an $H$-representation of $A$. If (4), (5) and (6) are satisfied, $A H A^{*} H^{-1}=D R H R^{*} D^{*} H^{-1}=D H D^{*} H^{-1}=D^{2}$. Further, if $A H A^{*} H^{-1}$ $=D^{2}$ and $D H=H D^{*}$, then (6) is satisfied with $R=D^{-1} A$. For $D^{-1} A H\left(D^{-1} A\right)^{*}=D^{-1} A H A^{*}\left(D^{-1}\right)^{*}=D^{-1} D^{2} H\left(D^{-1}\right)^{*}=H$ by Therefore we have proved this lemma.

LEMma 1. A necessary and sufficient condition that a matrix $A$ have an $H$-representation is that there exist a matrix $D$ such that $D H=H D^{*}$ and $A H A^{*} H^{-1}=D^{2}$.

If $A$ has the $H$-representation (4) and $Q A Q^{-1}=A_{1}, Q D Q^{-1}=D_{1}$, $Q R Q^{-1}=R_{1}$ and $Q H Q^{*}=H_{1}$, then $A_{1}=D_{1} R_{1}$ where $D_{1} H_{1}=H_{1} D_{1}{ }^{*}$ and $R_{1} H_{1} R_{1}{ }^{*}=H_{1}$. Moreover $H_{1}=Q H Q^{*}$ and $A_{1} H_{1} A_{1}{ }^{*}=Q A H A^{*} Q^{*}$, so that the two pencils of hermitian matrices $A H A^{*}-x H$ and $A_{1} H_{1} A_{1}{ }^{*}$ $-x H_{1}$ are conjunctive. Therefore, if $A$ has an $H$-representation and if the two pencils $A H A^{*}-x H$ and $A_{1} H_{1} A_{1}{ }^{*}-x H_{1}$ are conjunctive, the matrix $A_{1}$ has an $H_{1}$-representation. Accordingly we may suppose that the pencil $A H A^{*}-x H$ is in a canonical or normal form previously determined. ${ }^{2}$ Since $D H$ is hermitian, the elementary di-

${ }^{2} \mathrm{G}$. R. Trott, On the canonical form of a nonsingular pencil of hermitian matrices, American Journal of Mathematics, vol. 56 (1934), pp. 359-371. H. W. Turnbull, Pencils of hermitian forms, Proceedings of the London Mathematical Society (2), vol. 39 (1935), pp. 232-248. 
visors of $D-x E$ are the same as those of the pencil of hermitian matrices $D H-x H$. Complex elementary divisors of such a pencil must therefore occur in conjugate pairs and in particular this is true of the pure imaginary elementary divisors. Hence, if $D-x E$ has the elementary divisor $(x-i b)^{r}$ repeated $s$ times, where $b$ is real, then $D-x E$ also has the elementary divisor $(x+i b)^{r}$ repeated $s$ times and $D^{2}-x E$ has the elementary divisor $\left(x+b^{2}\right)^{r}$ repeated $2 s$ times. Since the elementary divisors of $D^{2}-x E$ are the same as those of $A H A^{*}$ $-x H$, the matrix $A$ cannot have an $H$-representation if the pencil $A H A^{*}-x H$ has an elementary divisor $\left(x+b^{2}\right)^{r}$, that is, a negative elementary divisor, repeated an odd number of times.

We proceed to determine what further conditions, if any, must be satisfied in order that $A$ may have an $H$-representation. In canonical form the matrices $H$ and $A H A^{*}$ are similarly partitioned diagonal block matrices of such a nature that it is only necessary to consider the three special cases in which the pencil $A H A^{*}-x H$ has

(i) only the two elementary divisors $(x-a)^{n},(x-\bar{a})^{n}, a \neq \bar{a}$,

(ii) the single elementary divisor $\left(x-b^{2}\right)^{n}, b$ real,

(iii) only the two elementary divisors $\left(x+b^{2}\right)^{n},\left(x+b^{2}\right)^{n}, b$ real.

Case (i). In canonical form

$$
H=\left(\begin{array}{ll}
0 & E \\
E & 0
\end{array}\right) \text { and } A H A^{*} H^{-1}=\left(\begin{array}{cc}
a(E+U) & 0 \\
0 & \bar{a}(E+U)^{\prime}
\end{array}\right),
$$

where $U$ is the auxiliary unit matrix of order $n$. Let

$$
\begin{aligned}
& G=(E+U)^{1 / 2}=E+U / 2-U^{2} / 8+\cdots+\alpha U^{n-1}, \\
& 2^{2 n-4}(n-3) !(n-1) ! \alpha=(-1)^{n}(2 n-5) !
\end{aligned}
$$

Then $G^{\prime}=\left(E+U^{\prime}\right)^{1 / 2}$. If $a=\rho^{2} e^{i \theta}, a^{1 / 2}=\delta \rho e^{i \theta / 2}$ and $\bar{a}^{1 / 2}=\delta \rho e^{-i \theta / 2}$, where $\delta= \pm 1$. Therefore, if $D=\left[a^{1 / 2} G, \bar{a}^{1 / 2} G^{\prime}\right], D H=H D^{*}$ and $D^{2}=A H A^{*} H^{-1}$. The signature of $D H$ is the same as that of $H$ since the signature of both matrices is zero. Since $\delta$ may have either of the values 1 or -1 , $D$ is not unique.

Case (ii). In canonical form

$$
H=\epsilon T \text { and } A H A^{*}=b^{2}(E+U) H,
$$

where $\epsilon= \pm 1$ and $T$ is the counter unit matrix, so that

$$
T^{2}=E \text { and } T U=U^{\prime} T .
$$

If $D=b G$, where $G$ is defined by (7), $D^{2}=A H A^{*} H^{-1}$ and $D H=H D^{*}$ by (8). If $n$ is even the signatures of $D H$ and $H$ are both zero and, if in $D H, b$ is replaced by $-b$ the signature of the resulting matrix is 
also zero. If $n$ is odd, the signature of $H$ is $\epsilon$ and that of $D H$ is $\pm \epsilon$ according as $b$ is positive or negative. Therefore, if $D H$ has the same signature as $H$ and $n$ is even, $D$ is not unique but, if $n$ is odd, $D$ is unique.

Case (iii). If the matrix $D$ exists the pencil $D H-x H$ has only the two elementary divisors $(x+i b)^{n},(x-i b)^{n}$ and we may take $D H-x H$ in the canonical form

$$
H=\left(\begin{array}{cc}
0 & E \\
E & 0
\end{array}\right), \quad D=\left(\begin{array}{cc}
i b G & 0 \\
0 & -i b G^{\prime}
\end{array}\right)
$$

where $G$ is defined by (7). Hence

and

$$
A H A^{*} H^{-1}=D^{2}=\left(\begin{array}{cc}
-b^{2}(E+U) & 0 \\
0 & -b^{2}\left(E+U^{\prime}\right)
\end{array}\right)
$$

$$
H=\left(\begin{array}{ll}
0 & E \\
E & 0
\end{array}\right)
$$

while the matrix $H$ may be transformed without disturbing $D^{2}$ into

$$
\left(\begin{array}{cc}
T & 0 \\
0 & -T
\end{array}\right)
$$

For a matrix pencil $A H A^{*}-x H$ with elementary divisors $\left(x+b^{2}\right)^{n}$, $\left(x+b^{2}\right)^{n}$ there are three distinct possible canonical forms in which $H$ is one of the matrices

$$
\left(\begin{array}{cc}
\epsilon_{1} T & 0 \\
0 & \epsilon_{2} T
\end{array}\right)
$$

$\epsilon_{1}=1, \epsilon_{2}=-1 ; \epsilon_{1}=\epsilon_{2}=1$ or $\epsilon_{1}=\epsilon_{2}=-1$. If we call $\epsilon_{1}$ and $\epsilon_{2}$ the indices associated with the elementary divisor $\left(x+b^{2}\right)^{n}$, we see that $A$ has an $H$-representation, if and only if one index is positive and the other is negative. Further, even when $A$ does have an $H$-representation, $D$ is not unique. Since the canonical form for the pencil $A H A^{*}-x H$ is diagonal block, the following theorem follows immediately from Lemma 1.

TheOREM 1. Let $H$ be any nonsingular hermitian matrix and $A$ be a nonsingular matrix. Then $A$ has an $H$-representation $A=D R$, if and only if the negative elementary divisors of the pencil $A H A^{*}-x H$ occur in pairs and exactly half of the indices associated with each negative elementary divisor are positive. The matrix, $D$, and therefore the matrix 
$R$, is unique, if and only if all elementary divisors of the pencil are positive and of odd order.

If $H^{2}=E, A=D H H R=S V$, where $S=D H$ and $V=H R$. Moreover $S=S^{*}$ and $V H V^{*}=H^{3}=H$. Hence we have as a corollary the following.

CoRollary 1. If $H^{2}=E$, the matrix $A$ can be written in the form $A=S V$, where $S$ is hermitian with the same signature as $H$ and, $V H V^{*}=H$, if and only if the conditions of Theorem 1 are satisfied.

The known theorem on the uniqueness of the polar components of the matrix $A$ in (1) is a particular case of Theorem 1 . For, if $H=E$, $A H A^{*}=A A^{*}$ and the elementary divisors of the pencil $A A^{*}-x E$ are all positive and linear.

3. Modified representation of any nonsingular matrix. Even if the matrix $A$ does not satisfy the conditions of Theorem 1 , it is possible to find a somewhat different representation of $A$. We first reduce the pencil $A H A^{*}-x H$ by conjunctive transformation to

$$
\left(\begin{array}{cc}
A_{1} H_{1} A_{1}^{*} & 0 \\
0 & A_{2} H_{2} A_{2}^{*}
\end{array}\right)-x\left(\begin{array}{cc}
H_{1} & 0 \\
0 & H_{2}
\end{array}\right),
$$

where no elementary divisor of $A_{1} H_{1} A_{1}-x H_{1}$ is negative and all elementary divisors of $A_{2} H_{2} A_{2}{ }^{*}-x H_{2}$ are negative. Then $A_{1}=D_{1} R_{1}$ and, since all elementary divisors of $-\mathrm{A}_{2} \mathrm{H}_{2} \mathrm{~A}_{2}{ }^{*}-x \mathrm{H}_{2}$ are positive, there exists a matrix $D_{2}$, such that $D_{2}^{2}=-A_{2} H_{2} A_{2}{ }^{*} H_{2}^{-1}$ and $D_{2} H_{2}$ $=H_{2} D_{2}{ }^{*}$. If $R_{2}=D_{2}^{-1} A_{2}$, then $R_{2} H_{2} R_{2}{ }^{*}=D_{2}^{-1} A_{2} H_{2} A_{2}{ }^{*}\left(D_{2}{ }^{*}\right)^{-1}=D_{2}$ - $\left(-D_{2}^{2} H_{2}\right)\left(D_{2}^{*}\right)^{-1}=-H_{2}$. Therefore $A_{2}=D_{2} R_{2}$, where $D_{2} H_{2}$ is hermitian with the same signature as $\mathrm{H}_{2}$ and $\mathrm{R}_{2} \mathrm{H}_{2} \mathrm{R}_{2}^{*}=-\mathrm{H}_{2}$. Let

$$
V=\left(\begin{array}{cc}
E_{1} & 0 \\
0 & -E_{2}
\end{array}\right)=\left[E_{1},-E_{2}\right],
$$

where $E_{i}$ is the unit matrix of the same order as $H_{i}$. Then $V^{2}=E$. If $A=\left[A_{1}, A_{2}\right], H=\left[H_{1}, H_{2}\right], R=\left[R_{1}, R_{2}\right]$ and $D=\left[D_{1}, D_{2}\right]$, then $A=D R$ where $D H$ is hermitian with the same signature as $H$ and $R H R^{*}=H V$, so that the signature of $H V$ is the same as that of $H$. Further from its form it is apparent that $V$ is a polynomial in $A H A^{*} H^{-1}$ and that $V H=H V=H V^{*}$, so that $V H V^{*}=H$. While we have used a special form of the pencil $A H A^{*}-x H$ it follows that similar results are true when the pencil is not in this form. Accordingly we have proved this theorem.

TheOREM 2. Let $H$ be a nonsingular hermitian matrix and let $A$ be 
a nonsingular matrix of the same order as $H$. Then there exists at least one matrix $V$, a polynomial in $A H A^{*} H^{-1}$, with the properties that $V^{2}=E, V H$ is hermitian with the same signature as $H$ and that no characteristic number of $A H A^{*}(V H)^{-1}$ is negative. The matrix $A=D R$ where $D H$ is hermitian with the same signature as $H$ and $R H R^{*}=V H$. If all the characteristic numbers of $A H A^{*} H^{-1}$ are real, the matrix $V$ is uniquely determined.

4. Representation in the real field. If $H$ is a real symmetric matrix and $A$ a real nonsingular matrix our argument may be carried through in the field of real numbers with only a few alterations. In (i), if the pair of conjugate elementary divisors is $(x-c \pm i d)^{n}$, we take $H=q T, A H A^{*}=p(E+U) q T$, where

$$
p=\left(\begin{array}{cc}
c & d \\
-d & c
\end{array}\right) \text { and } q=\left(\begin{array}{ll}
0 & 1 \\
1 & 0
\end{array}\right)
$$

Then, if

$$
\begin{aligned}
& \left(\begin{array}{cc}
r & s \\
-s & r
\end{array}\right)^{2}=p, \\
& \left(\begin{array}{cc}
r & s \\
-s & r
\end{array}\right)^{2} G H
\end{aligned}
$$

is symmetric and

$$
\left\{\left(\begin{array}{cc}
r & s \\
-s & r
\end{array}\right) G\right\}^{2}=A H A^{*} H^{-1} \text {. }
$$

Case (ii) is unaltered. In (iii) we replace the matrices $D$ and $H$ of (9) by

$$
\left(\begin{array}{cc}
0 & b G \\
-b G & 0
\end{array}\right) \text { and }\left(\begin{array}{cc}
0 & T \\
T & 0
\end{array}\right)
$$

respectively. It therefore follows that Theorems 1 and 2 are true in the field of real numbers, if hermitian is replaced by symmetric and conjugate transposed by transposed.

5. Conditions for the commutativity of the polar components. Let the matrix $A$ have an $H$-representation $A=D R$. Then $A=R D_{1}$ where $D_{1}=R^{-1} D R$. The matrix $D_{1} H$ is hermitian. Further $H A^{*} H^{-1} A$ $=H D_{1}^{*} R^{*} H^{-1} R D_{1}=D_{1} H H^{-1} D_{1}=D_{1}^{2}$. Since $D^{2}=A H A^{*} H^{-1}$, it follows that, if $A^{*}$ is commutative with $H^{-1} A H, D_{1}^{2}=D^{2}$ and that for a proper choice $D_{1}=D$. Conversely, if $D_{1}=D, H^{-1} A H$ is commutative with $A^{*}$. We have therefore proved this theorem. 
Theorem 3. If $A$ has an $H$-representation $A=D R, A$ also has an $H$-representation $A=R D_{1} . A$ necessary and sufficient condition that $D=D_{1}$ or that $D$ be commutative with $R$ is that $A^{*}$ be commutative with $H^{-1} A H$.

Therefore, if $A=D R$ and $A$ is normal ${ }^{3}$ with respect to $H, A=R D$ so that $R$ and $D$ are commutative. That the converse is not true may be shown as follows. Let

$$
H=\left(\begin{array}{llll}
0 & 0 & 1 & 0 \\
0 & 0 & 0 & 1 \\
1 & 0 & 0 & 0 \\
0 & 1 & 0 & 0
\end{array}\right) \text { and } A=\left(\begin{array}{cccc}
1 & 0 & 0 & 0 \\
0 & 1 & 0 & 0 \\
0 & 0 & 1 & 0 \\
0 & 0 & 1 & 1
\end{array}\right)
$$

Then

$$
A H A^{*} H^{-1}=\left(\begin{array}{llll}
0 & 0 & 1 & 0 \\
0 & 0 & 0 & 1 \\
1 & 0 & 0 & 0 \\
1 & 1 & 0 & 0
\end{array}\right)\left(\begin{array}{llll}
0 & 0 & 1 & 0 \\
0 & 0 & 0 & 1 \\
1 & 1 & 0 & 0 \\
0 & 1 & 0 & 0
\end{array}\right)=\left(\begin{array}{llll}
1 & 1 & 0 & 0 \\
0 & 1 & 0 & 0 \\
0 & 0 & 1 & 0 \\
0 & 0 & 1 & 1
\end{array}\right) .
$$

The characteristic numbers of $A H A^{*} H^{-1}$ are all plus one and therefore $A$ has an $H$-representation. In fact

$$
D=\left(\begin{array}{cccc}
1 & 1 / 2 & 0 & 0 \\
0 & 1 & 0 & 0 \\
0 & 0 & 1 & 0 \\
0 & 0 & 1 / 2 & 1
\end{array}\right) \quad \text { and } R=\left(\begin{array}{cccc}
1 & -1 / 2 & 0 & 0 \\
0 & 1 & 0 & 0 \\
0 & 0 & 1 & 0 \\
0 & 0 & 1 / 2 & 1
\end{array}\right)
$$

Hence

$$
H^{-1} A H=\left(\begin{array}{cccc}
1 & 0 & 0 & 0 \\
1 & 1 & 0 & 0 \\
0 & 0 & 1 & 0 \\
0 & 0 & 0 & 1
\end{array}\right)
$$

and is commutative with $A^{*}$. However, $H^{-1} A H$ is not a polynomial in $A^{*}$ and is therefore not normal with respect to $H$.

If the elementary divisors of $A H A^{*}-x H$ are all positive and linear and if $A=D R=R D$, it is comparatively easy to show that $H^{-1} A H$ is a polynomial in $A^{*}$ and therefore that $A$ is normal with respect to $H$.

${ }^{3}$ John Williamson, Matrices normal with respect to an hermitian matrix, American Journal of Mathematics, vol. 60 (1938), pp. 355-373. 
This seems to be the generalization of the classical result that a necessary and sufficient condition for the polar components of a matrix $A$ to be commutative is that $A$ be a normal matrix.

Queens College

\section{REMARKS ON REGULARITY OF METHODS OF SUMMATION}

\section{G. E. FORSYTHE AND A. C. SCHAEFFER}

A doubly infinite matrix ${ }^{1}\left(a_{m n}\right)(m, n=1,2, \cdots)$ is said to be regu$l a r$, if for every sequence $x=\left\{x_{n}\right\}$ with limit $x^{\prime}$ the corresponding sums $y_{m}=\sum_{n=1}^{\infty} a_{m n} x_{n}$ exist for $m=1,2, \cdots$, and if $\lim _{m \rightarrow \infty} y_{m}=x^{\prime}$. An apparently more inclusive definition of regularity is that for each sequence $x$ with limit $x^{\prime}$ the sums defining $y_{m}$ shall exist for all $m \geqq m_{0}(x)$ and $\lim _{m \rightarrow \infty} y_{m}=x^{\prime}$. Tamarkin 2 has shown that $\left(a_{m n}\right)$ is regular in the latter sense if and only if there exists an $m_{1}$ independent of $x$ such that the matrix $\left(a_{m n}\right)\left(m \geqq m_{1}, n \geqq 1\right)$ is regular in the former sense. Using point set theory in the Banach space $(c)$, he proves a theorem ${ }^{3}$ from which follows the result just mentioned. This note presents an elementary proof of that theorem and discusses some related topics.

THEOREM 1. Suppose the doubly infinite matrix $\left(a_{m n}\right)$ has the property that for each sequence $x=\left\{x_{n}\right\}$ with limit 0 there exists an $m_{0}=m_{0}(x)$ such that for all $m \geqq m_{0}(x), u_{m}=\lim \sup _{k \rightarrow \infty}\left|\sum_{n=1}^{k} a_{m n} x_{n}\right|<\infty$. Then there exists an $m_{1}$ such that $\sum_{n=1}^{\infty}\left|a_{m n}\right|<\infty$ for all $m \geqq m_{1}$.

If in addition $\lim _{m \rightarrow \infty} u_{m}=0$ for each sequence $x$ with limit 0 , it will follow ${ }^{4}$ that there exists an $N$ such that $\sum_{n=1}^{\infty}\left|a_{m n}\right| \leqq N<\infty$, for all $m \geqq m_{1}$.

To prove Theorem 1, suppose there were an infinite sequence $m_{1}<m_{2}<\cdots$ such that $\sum_{n=1}^{\infty}\left|a_{m n}\right|=\infty$ for $m \in\left\{m_{\nu}\right\}$. Let $x_{1}, \cdots, x_{k_{1}}$ be chosen with unit moduli and with amplitudes such that

Presented to the Society, April 11, 1942 under the title $A$ remark on Toeplitz matrices; received by the editors January 22, 1942.

${ }^{1}$ In this note $a_{m n}, x_{n}$ and $x^{\prime}$ denote finite complex numbers.

${ }^{2} \mathrm{~J}$. D. Tamarkin, On the notion of regularity of methods of summation of infinite series, this Bulletin, vol. 41 (1935), pp. 241-243.

${ }^{3}$ J. D. Tamarkin, loc. cit., p. 242, lines 1-6.

${ }^{4}$ See, for example, I. Schur, Über lineare Transformationen in der Theorie der unendlichen Reihen, Journal für die reine und angewandte Mathematik, vol. 151 (1921), pp. 79-111; p. 85, Theorem 4. 